\title{
Analytical Solution of Safety Stock Determination in Case of Uncertain Unimodal Lead-Time Demand
}

\author{
Gerrit K. Janssens ${ }^{1}$, Lotte Verdonck ${ }^{2}$, Katrien Ramaekers ${ }^{3}$ \\ ${ }^{1-3}$ Hasselt University, Hasselt, Belgium
}

\begin{abstract}
As companies state that a delivery service is important to their customers, an out-of-stock is considered harmful and therefore they keep safety stock in case of uncertain demand. For decision making on the level of safety stock a complete formulation of the distributional form of the demand during lead time is required. In practice, this information may not be available. In such a case, only partial information on the distribution might be available, such as the range, the mode, the mean or the variance. Given a value for a service performance measure, the decision maker, in this case, is not confronted with a single value for the safety stock but rather with an interval. The present research shows how upper and lower bounds of the safety stock are obtained in an analytical way, given a pre-specified service level using a service performance measure, called 'expected number of units short'. The technique is also illustrated and compared within the framework of the research.
\end{abstract}

Keywords - Inventory management, uncertain demand, safety stock, unimodal distributions.

\section{INTRODUCTION}

In the business world, investment in inventories might be very high. Furthermore, companies are confronted with fluctuations in inventories in time and with uncertainties both in demand and supply, which directly influence decisions on inventories. Logistics managers use sophisticated systems for inventory management that enable them to take correct and timely decisions. Software implements decision models, which concentrate on the determination of inventory replenishment quantities based on relevant costs, such as order costs or storage costs. Most of these models assume deterministic demand patterns. But in real life uncertainties may appear both in demand and in supply or even in the quality of the delivered goods. The present research investigates in detail a specific case of inventory management decisions in the case of demand uncertainty.

The decisions are made on the basis of optimisation models taking a performance measure into consideration, which might be cost-oriented or service-oriented. Performance measures of the service-oriented type may be expressed relatively as a probability of a stock-out during a certain replenishment period, or may be expressed absolutely in terms of the number of units short, which is a direct indication for lost sales. These types of measures do not explicitly include a cost for additional paperwork or transport cost or loss of goodwill as, in practice, experience shows that these costs are hard to determine. Both in the cost-oriented and in the service-oriented performance evaluation, a specific integral plays an important role in the decision-making process. This integral is defined by Silver et al. [1, p. 258] as the expected shortage per replenishment cycle (ESPRC):

$$
E S P R C=\int_{t}^{+\infty}(x-t) f(x) d x,
$$

in which it is assumed that the demand $x$ in a replenishment lead time has a probability density function $f(x)$ and an order is placed at some time when the inventory position is at level $t$. If ordered per quantity $Q$, the fraction backordered is equal to $E S P R C / Q$ and a performance measure, indicated as $P_{2}$, is defined as (see [1]):

$$
P_{2}=1-E S P R C / Q \text {. }
$$

In classical textbooks, the decision on the level of safety stock is based on the expected value and the standard deviation of the lead-time demand. Furthermore, it is assumed that the demand follows a Normal distribution, so that, given a service level and the knowledge about the first two moments, the required safety stock can be determined. However, normality of the distribution is sometimes violated. Assuming normality, when there is not true distribution behind the data, it might lead to either a degraded service level or an increase in the storage cost.

Regarding the violation of the normality assumption, the scientific literature is contradictory. Naddor [2] finds that false assumptions about the distribution may lead to higher cost in the case of extreme distributions, but, with realistic distributions, only the first and second moments are essential. On the other hand, Bartezzaghi et al. [3] show a significant impact of the shape of the demand distribution on the service level, based on a large set of experiments. Their analysis demonstrates that the shape of the distribution is a primary factor in the determination of inventories. Lau and Zaki [4] note that mean and variance are not sufficient for safety stock calculation, since skewness and kurtosis should also be accounted for. Furthermore, Käki et al. [5] show the impact of the demand distribution shape on replenishment, based on experiments with qualitative shape characteristics (normal, positively skewed, negatively skewed, and bimodal). In Janssens and Ramaekers' research [6], an approach has been developed to obtain the reorder point based on the knowledge of the range, mean and variance of the demand distribution only, which is the same information as required for the use of the normal distribution (as many times used in commercial software).

Given a mean and a standard deviation, and without knowledge of the real probability distribution behind the data, many distributions are candidate distributions. Each of them would lead to a different safety stock, given a preset service 
level. A conservative approach would be to determine the safety stock in the worst case distribution. In case only partial information on the probability density function is available (such as range, mean or variance), it is possible to determine the worst-case distribution. The mathematical development, however, shows that the worst-case distributions (in case mean and variance are known) are discrete distributions, in which the mass is concentrated in two points. This property has already been described by Scarf [7].

It might be argued that this approach is too conservative for practical purposes. Let us think of convincing a logistics manager to determine the safety of stock of a specific product based on the idea that the demand distribution has mass only in two points. A manager certainly will agree that some probability distribution, including uncertainty, has to be taken into account, but the distribution does not have extreme shapes. A manager can believe in asymmetry of the distribution, but it is rather uncommon that the manager will believe that the distribution is not unimodal. This means that the 'conservative' approach should be modified to a less conservative one, in which the 'worst case' distributions have a unimodal shape.

The present paper aims at investigating how such a distribution can be determined and which techniques are available to achieve it. It would be outstanding if a set of analytical formulas were available to determine the safety stock, given the knowledge of range, mean and variance of the demand during lead time, with the constraint that the resulting distribution should be unimodal. However, up to now, this simple solution is not available and other methods need to be explored. In the following section, solution methods first are explored in the case of knowledge of range, mean and variance but without constraints on unimodality. Afterwards, it is investigated which of these methods can be used with the constraint of unimodality. Illustrations, opportunities and limitations of the use of the methods are provided for the unimodal case.

\section{SOLUTIONS FOR THE CASE WITHOUT CONSTRAINT ON UNIMODALITY}

In this section, the incomplete information on the demand during lead time includes the finite range of the distribution, and the first and second moments. Let the size of the demand $X$ for a specific product in a finite period have a distribution $F$ with first two moments $\mu_{1}=E(X)$ and $\mu_{2}=E\left(X^{2}\right)$. The distribution $F$ is defined on the finite interval $[a, b]$.

From a mathematical point of view, the problem is to find the following bounds:

$$
\sup _{F \in \phi} \int_{0}^{\infty}(x-t)_{+} d F(x),
$$

where $\phi$ is the class of all distribution functions $F$ which have moments $\mu_{1}$ and $\mu_{2}$, and which have support in $\mathfrak{R}^{+}$.
Let further $\sigma^{2}=\mu_{2}-\mu_{1}^{2}$. We assume $t$ to be strictly positive.

The domain of the parameters is

$$
\begin{aligned}
& a \leq \mu_{1} \leq b, \quad 0 \leq \sigma^{2} \leq\left(\mu_{1}-a\right)\left(b-\mu_{1}\right) \text { or } \\
& \mu_{1}^{2} \leq \mu_{2} \leq \mu_{1}(a+b)-a b
\end{aligned}
$$

Further the following abbreviations are used: $\sigma_{\mu t}^{2}=\sigma^{2}+\left(\mu_{1}-t\right)^{2}$ and $c=1 / 2(a+b)$.

It has been shown that the upper bound corresponds to a 2atomic distribution, i.e., the probability mass is concentrated in two points (see Heijnen and Goovaerts [8] and Janssen, Haezendonck and Goovaerts [9]). Given a reorder point, the upper bound on the expected number of units short is given in Table I.

TABLE I

UPPER BOUNDS FOR THE EXPECTED NUMBER OF UNITS SHORT

\begin{tabular}{|c|c|c|}
\hline \multicolumn{1}{|c|}{ Conditions } & Upper bounds & $\begin{array}{c}\text { Atoms of } \\
\text { solution }\end{array}$ \\
\hline$t \leq c, \sigma_{\mu t} \leq t-a$ & $1 / 2\left(\sigma_{\mu t}+\mu_{1}+t\right)$ & $t-\sigma_{\mu t}, t+\sigma_{\mu t}$ \\
\hline$t \leq c, \sigma_{\mu t} \geq t-a$ & $\left(\mu_{1}-a\right) \frac{\left(\mu_{1}-t\right)\left(\mu_{1}-a\right)+\sigma^{2}}{\left(\mu_{1}-a\right)^{2}+\sigma^{2}}$ & $a, \mu_{1}+\frac{\sigma^{2}}{\mu_{1}-a}$ \\
\hline$t \geq c, \sigma_{\mu t} \leq b-t$ & $1 / 2\left(\sigma_{\mu t}+\mu_{1}+t\right)$ & $t-\sigma_{\mu t}, t+\sigma_{\mu t}$ \\
\hline$t \geq c, \sigma_{\mu t} \geq b-t$ & $\frac{(b-t) \sigma^{2}}{\left(b-\mu_{1}\right)^{2}+\sigma^{2}}$ & $\mu_{1}-\frac{\sigma^{2}}{b-\mu_{1}}, b$ \\
\hline
\end{tabular}

While Table I has all ingredients to determine the reorder point (and by this the safety stock), the decision problem is different from what the table offers. The table provides the upper bound on the expected number of units short given a value of $t$, but the decision problem is looking for a value of $t$, given a maximum value of the expected number of units short. There are several solutions proposed to solve the decision problem:

- if an analytical equation exists, the equation for $t$ should be solved in an analytical or numerical way (Method 1);

- $\quad$ if an analytical solution exists, the equation should be solved by a one-dimensional search procedure (Method 2);

- if no analytical procedure exists, a linear program should be constructed to find an approximative solution and use a one-dimensional search procedure (Method 3).

As can be noticed from Table I, the equations (listed in the second column called 'Upper bounds') are non-linear but not hard non-linear functions. Thus, for this illustrative example, the first of the three solution methods is used. This means, as the conditions (in the first column) cannot be checked in 
advance, all four equations need to be solved and the solutions need to be validated with the conditions. The conditions are mutually exclusive, so no confusion can exist. The solutions can be found by any non-linear equation solver, including spreadsheets like Microsoft Excel, using the Solver. Let us illustrate this way of working by the following example.

The parameters of the problem are: $a=0, b=50, \mu_{1}=30$, $\mu_{2}=1200$, so $c=25, \sigma^{2}=300$ and $\sigma_{\mu t}^{2}=325$. Let us put the upper bound of the expected number of units short equal to 12 . The solutions of the four equations are listed in Table II.

TABLE II

SOLUTION BY METHOD 1

\begin{tabular}{|c|c|c|}
\hline Condition & Solution & Validity \\
\hline$t \leq c, \sigma_{\mu t} \leq t-a$ & 24.02 & Yes \\
\hline$t \leq c, \sigma_{\mu t} \geq t-a$ & 24.00 & No \\
\hline$t \geq c, \sigma_{\mu t} \leq b-t$ & 24.03 & No \\
\hline$t \geq c, \sigma_{\mu t} \geq b-t$ & No solution & No \\
\hline
\end{tabular}

The function, describing the upper bound, is a decreasing function in $t$. That makes it easy to apply Method 2, given that $t=0$ offers the expected value (30 in the example) and $t=50$ offers zero. The procedure of cutting the relevant range into halves offers an efficient way of finding the solution. The pseudocode of the procedure is shown below. Target refers to the required value of the expected number of units short. Required_precision refers to the precision required on the value of $t$. Running $t$ refers to the running value of $t$ as it changes during the procedure. Precision refers to the running value of the precision, calculated as the difference between the previous and current value of upper bound. Upper bound refers to the value of the upper bound as calculated from Table I. Interval refers to the size of the interval, which is used for adapting the value of $t$.

Parameters target, precision_required, $a, b$

interval $:=(b-a) / 2$

running $t:=a+$ interval

precision $:=b-a$

while precision $\geq$ precision_required do

case

condition $t \leq c, \sigma_{\mu t} \leq t-a$ :

$$
\text { upper_bound }:=1 / 2\left(\sigma_{\mu t}+\mu_{1}+t\right)
$$

condition $t \leq c, \sigma_{\mu t} \geq t-a$

$$
\text { upper_bound }:=\left(\mu_{1}-a\right) \frac{\left(\mu_{1}-t\right)\left(\mu_{1}-a\right)+\sigma^{2}}{\left(\mu_{1}-a\right)^{2}+\sigma^{2}}
$$

condition $t \geq c, \sigma_{\mu t} \leq b-t$

$$
\text { upper_bound }:=1 / 2\left(\sigma_{\mu t}+\mu_{1}+t\right)
$$

condition $t \geq c, \sigma_{\mu t} \geq b-t$

$$
\text { upper_bound }:=\frac{(b-t) \sigma^{2}}{\left(b-\mu_{1}\right)^{2}+\sigma^{2}}
$$

endcase

interval $:=$ interval / 2

precision $:=\operatorname{abs}($ upper_bound - target)

if upper_bound $\leq$ target

running_t $t=$ running_t $t$-interval

else

running_t $:=$ running_t $t+$ interval

endif

endwhile

The example is worked out in Table III. A number of steps in the procedural computations are shown. The procedure stops whenever the required precision is obtained.

TABLE III

SOLUTION BY METHOD 2

\begin{tabular}{|l|l|l|l|}
\hline \multicolumn{1}{|c|}{$t$} & $\begin{array}{l}\text { Upper } \\
\text { bound }\end{array}$ & \multicolumn{1}{c|}{$t$} & $\begin{array}{c}\text { Upper } \\
\text { bound }\end{array}$ \\
\hline 25 & 11.51 & 23.4375 & 12.54 \\
\hline 12.5 & 21.06 & 24.21875 & 12.02 \\
\hline 18.75 & 15.94 & 24.609375 & 11.76 \\
\hline 21.875 & 13.63 & 24.414063 & 11.89 \\
\hline
\end{tabular}

The third method will be illustrated in the unimodal case (in section III)

\section{SOLUTIONS FOR THE CASE WITH A CONSTRAINT ON UNIMODALITY}

Sometimes it is considered difficult to observe or to estimate both first and second moments, but experts have an opinion on the unimodality of a demand distribution, either with or without additional knowledge on the expected value. This idea has been common in expert opinion on the duration of activities in a project, where experts estimate three values: an optimistic, a most likely (or modal) and a pessimistic duration [10].

Let $I$ be an interval on the real line $\mathfrak{R}$. A fixed point $m \in I$ is called the mode. A m-unimodal density function is a density function increasing (not necessarily strict) at the left of $m$ and decreasing (not necessarily strict) at the right of $m$. Let us consider first the case in which the range, expected value and mode are known. The domain of the parameters is: $a \leq m \leq b, \frac{1}{2}(a+m) \leq \mu \leq \frac{1}{2}(b+m)$. In this case, analytical upper bounds exist [11, Chapter 5]. The bounds are shown in Table IV.

TABLE IV

UPPER BOUNDS FOR THE EXPECTED NUMBER OF UNITS SHORT IN THE UNIMODAL CASE

\begin{tabular}{|c|c|}
\hline Condition & Solution \\
\hline$m \leq t$ & $\left(\mu_{1}-\frac{a+m}{2}\right) \frac{(b-t)^{2}}{(b-m)(b-a)}$ \\
\hline$m \geq t$ & $\frac{1}{2} \frac{(b-t)^{2}}{b-a}+\left(\mu_{1}-c\right)\left(1-\frac{(t-a)^{2}}{(b-a)(m-a)}\right)$ \\
\hline
\end{tabular}


Method 1 is also applicable in this case. With the same data (but no variance known), let us add the additional information of the mode $m=10$. The result of the application of Method 1 is shown in Table $\mathrm{V}$.

TABLE V

SOLUTION BY METHOD 1

\begin{tabular}{|c|c|c|}
\hline Condition & Solution & Validity \\
\hline$m \leq t$ & 19.02 & Yes \\
\hline$m \geq t$ & 18.00 & No \\
\hline
\end{tabular}

The type of distributions that correspond to the extreme distributions leading to the upper bound are distributions, which consist of rectangular parts. The probability density in the left part (between 0 and the mode on the $x$-axis) is smaller than the density on the right part (between the mode and the upper bound of the range). In the example, the probability density is given by $f(x)=0$ if $0 \leq x \leq 10$ and $f(x)=1 / 40$ if $10<x \leq 50$.

Method 2 is also applicable in the case of unimodal distributions with a known mode. In Table VI, a number of steps in the procedural computations are shown. The procedure is similar to the pseudocode as presented in Section III: the 'case' component has only two choices, i.e., related to the conditions mentioned in Table IV.

TABLE VI

SOLUTION BY METHOD 2

\begin{tabular}{|l|l|l|l|}
\hline \multicolumn{1}{|c|}{$t$} & \multicolumn{1}{|c|}{$\begin{array}{c}\text { Upper } \\
\text { bound }\end{array}$} & \multicolumn{1}{c|}{$t$} & $\begin{array}{c}\text { Upper } \\
\text { bound }\end{array}$ \\
\hline 25 & 7.8125 & 20.3125 & 11.01685 \\
\hline 12.5 & 17.57813 & 19.53125 & 11.60431 \\
\hline 18.75 & 12.20703 & 18.945313 & 12.05492 \\
\hline 21.875 & 9.887695 & 19.04297 & 11.97922 \\
\hline
\end{tabular}

In case, more constraints are involved, it is hardly possible to find analytical solutions and, therefore, to find the way to obtain the best value of safety stock or reorder point. In this situation, the authors of the present paper propose using a search procedure based on a linear programming approach. This corresponds to Method 3 mentioned in Section I. The method is elaborated and illustrated for the case of a unimodal distribution with mode known but, additionally to the knowledge of the expected value, also the constraint of a known variance is included. A separate section is dedicated to this topic.

\section{LineAR PROGRAMMING SOLUTION FOR THE CASE WITH A CONSTRAINT ON UNIMODALITY}

Let $f_{1}, f_{2}, \ldots, f_{n}$ be functions on $\mathfrak{R}$. For any $z^{\prime}=\left(z_{1}, \ldots, z_{n-1}\right) \in \mathfrak{R}^{n-1}, \quad$ we consider the primal maximisation problem:

$$
P\left(z^{\prime}\right)=\sup _{F \in \phi}\left[\int_{0}^{\infty}(x-t)_{+} d F(x) \mid I(F)\right],
$$

where $I(F)$ is a set of integral equality constraints of the type $\int f_{i}(x) d F(x)=z_{i},(i=1, \ldots, n-1)$ and $f_{n}=(x-t)_{+}$.

In our application, the constraints are moment constraints, i.e., the first and second moment equalities and the obvious constraint because any member of $\phi$ is a probability distribution.

$$
\int d F(x)=1, \int x d F(x)=\mu_{1}, \int x^{2} d F(x)=\mu_{2},
$$

which means that:

$$
\begin{aligned}
& n=3 \\
& f_{1}(x)=x \\
& f_{2}(x)=x^{2} \\
& f_{3}(x)=(x-t)_{+} \\
& z_{1}=\mu_{1} \\
& z_{2}=\mu_{2}
\end{aligned}
$$

First, let us introduce the method for the case of the problem with two moments known. The extreme distributions for the supremum problem has been shown to be a two-point distribution. The integral (4) may be approximated by a sum making use of finite masses $p_{i}$ in a large number of points $x_{i}$. Its formulation looks like this:

$$
\operatorname{Max} \sum_{i}\left(x_{i}-t\right)_{+} \cdot p_{i}
$$

subject to

$$
\begin{aligned}
& \sum_{i} p_{i}=1, \\
& \sum_{i} x_{i} \cdot p_{i}=\mu_{1} \\
& \sum_{i} x_{i}^{2} \cdot p_{i}=\mu_{2},
\end{aligned}
$$

and $p_{i} \geq 0, a \leq x_{i} \leq b$. However, any refinement in granularity (more values of $x_{i}$ ) leads to an increased number of variables both in the objective function (5) and in the constraints (6)-(8). This phenomenon does not guarantee any convergence towards the exact upper bound.

The optimisation problem (4) has a dual program of the type:

$$
Q\left(z^{\prime}\right)=\inf \left(\begin{array}{c}
\wedge \\
y_{1} m_{1}+y_{2} m_{2}+y_{3} \mid y_{1} \hat{f_{1}}(\theta)+y_{2} f_{2}(\theta)+y_{3} \geq f_{3}(\theta)
\end{array}\right)
$$

$(\theta \in J)$,

where the infimum is over all $y=\left(y_{1}, y_{2}, y_{3}\right) \in \mathfrak{R}^{3}$ satisfying the constraints indicated after the slash and $J$ is a nonvoid subset of $I$. The functions $f_{i}(\theta)(i=1, \ldots, n)$ are defined on $J$. In the situation for the family of distributions on 
a finite interval $[0, b]$, where $b$ is a fixed positive number, the three functions are:

$$
\begin{array}{lll}
\wedge & \wedge & \wedge \\
f_{1}(\theta)=\theta, & f_{2}(\theta)=\theta^{2}, & f_{3}(\theta)=(x-\theta)_{+}
\end{array}
$$

Mostly the set $J$ is infinite, so the number of linear constraints on $y$ is infinite. The idea is to replace $J$ with a large finite subset of $J$ and then to solve the obtained linear program. Through a careful selection of the $\theta$-values, convergence to the supremum is guaranteed as exemplified by Janssens \& Ramaekers (2008). The optimisation problem (9) can thus be approximated by the problem $Q^{A}$ :

$$
\begin{aligned}
& Q^{A}\left(z^{\prime}\right)=\inf _{y \in \Re^{3}}\left(y_{1} m_{1}+y_{2} m_{2}+y_{3} \mid y_{1} \theta_{i}+y_{2} \theta_{i}^{2}+y_{3} \geq\left(\theta_{j}-t\right)_{+}\right), \\
& \left.\left(\theta_{j}=j^{*} \frac{b}{k}, j=0, \ldots, k\right)\right) .
\end{aligned}
$$

A numerical example demonstrates the use of bounds on the expected number of units short. The following information on demand during the replenishment period is known: the first moment $\mu_{1}=25$, the second moment $\mu_{2}=725$ and the range of demand is $[0,50]$. The upper bounds on the number of stockout units are presented in Table VII for different values of $t$ and varying sizes $k$ of the evaluation point set. The exact values for the upper bounds are 16.37931 for $t=10,5.0$ for $t=25$ and 1.37931 for $t=40$.

TABLE VII

SOLUTIONS OF THE LP APPROXIMATION METHOD

\begin{tabular}{|c|c|c|c|c|}
\hline & $k=10$ & $k=20$ & $k=40$ & $k=80$ \\
\hline$t=10$ & 16.3333 & 16.3636 & 16.3768 & 16.3784 \\
\hline$t=25$ & 5.0000 & 5.0000 & 5.0000 & 5.0000 \\
\hline$t=40$ & 1.3333 & 1.3636 & 1.3768 & 1.3784 \\
\hline
\end{tabular}

In

case the constraint of a unimodal distribution with a known mode $m$ is introduced, the functions $\hat{f}($.$) take a different form:$

$$
\begin{gathered}
\widehat{f}_{1}=\frac{1}{2}(\theta+m) \\
\widehat{f_{2}}=\frac{1}{3}\left(m^{2}+m \theta+\theta^{2}\right) \\
\widehat{f_{3}}=\frac{1}{2} \frac{(\theta-t)_{+}^{2}}{\theta-m}
\end{gathered}
$$

With the same data, and additional values for the mode $m$, the problem (9) can be formulated. The upper bounds on the expected number of units short are presented in Table VIII.
TABLE VIII

SOLUTIONS OF THE LP APPROXIMATION METHOD: UNIMODAL CASE

\begin{tabular}{|l|c|c|c|c|}
\hline & $k=10$ & $k=20$ & $k=40$ & $k=80$ \\
\hline$t=10(m=5)$ & 17.7778 & 17.7778 & 17.7778 & 17.7778 \\
\hline$t=25(m=15)$ & 8.9286 & 8.9286 & 8.9286 & 8.9286 \\
\hline
\end{tabular}

Note that the number of constraints is linear in terms of the granularity $k$. This keeps the solution effort of the linear programs tractable. From the tables, it can be seen that with a reasonable value of $k$ a very good approximation can be obtained. The effort is higher than the one required for Methods 1 and 2. But for these methods an analytical solution of the integral, given the value of $t$, is required.

\section{CONCLUSION}

The research aims at proposing methods for obtaining a safety stock, given a customer service level, in a situation where the demand during lead time is not completely known. The incomplete information may include the range, first and second moments, unique mode, or tail probability. In some special cases, the expected number of units short can be expressed in an analytical way. In these cases, the incomplete information can be used to obtain an upper bound on the safety stock given a pre-described service level. Two methods are explained in the case of two moments known and in the case with a unique mode and the mean known. In most other cases, no analytical formulae exist on which our methods are based. For these situations, a linear programming approach is presented. The situation is more complex, but also leads to a good approximation of the required solution.

\section{REFERENCES}

[1] E. A. Silver, D. F. Pyke and R. Peterson, Inventory management and production planning and scheduling (third edition). New York, NY, USA: Wiley and Sons, 1998.

[2] E. Naddor, "Note-Sensitivity to distributions in Inventory Systems", Management Science, vol. 24, no. 16, pp. 1769-1772, 1978. https://doi.org/10.1287/mnsc.24.16.1769

[3] E. Bartezzaghi, R. Verganti, and G. Zotteri, "Measuring the impact of asymmetric demand distributions on inventories", International Journal of Production Economics, vol. 60-61, pp. 395-404, 1999. https://doi.org/10.1016/S0925-5273(98)00193-5

[4] H.-S. Lau and A. Zaki, "The sensitivity of inventory decisions to the shape of lead time-demand distribution", AIIE Transactions, vol. 14, no. 4, pp. 265-271, 1982. https://doi.org/10.1080/05695558208975239

[5] A. Käki, A. Salo, and S. Talluri, "Impact of the shape of demand distribution in decision models for operations management", Computers in Industry, vol. 64, no. 7, pp. 765-775, 2013. https://doi.org/10.1016/j.compind.2013.04.010

[6] G. K. Janssens and K. Ramaekers, "A linear programming formulation for an inventory management decision problem with a service constraint", Expert Systems with Application, vol. 38, no. 7, pp. 7929-7934, 2011. https://doi.org/10.1016/j.eswa.2010.12.009

[7] H. Scarf, "A min-max solution of an inventory problem." In K.J Arrow, S. Karlin and H. Scarf (Eds.) Studies in the mathematical methods of inventory and production. Redwood City, CA, Stanford University Press, pp. 201-209, 1958.

[8] B. Heijnen and M. J. Goovaerts, "Best upper bounds on risks altered by deductibles under incomplete information", Scandinavian Actuarial Journal, vol. 1989, no. 1, pp. 23-46, 1989. https://doi.org/10.1080/03461238.1989.10413853

[9] K. Janssen, J. Haezendonck and M. J. Goovaerts, "Upper bounds on stoploss premiums in case of known moments up to the fourth order", Insurance Mathematics and Economics, vol. 5, no. 4, pp. 315-334, 1986. https://doi.org/10.1016/0167-6687(86)90027-2 
[10] D. G. Malcolm, J. H. Roseboom, C. E. Clark and W. Fazar, "Application of a technique for research and development program evaluation", Operations Research, vol. 7, no. 5, pp. 646-669, 1959. https://doi.org/10.1287/opre.7.5.646

[11] M. J. Goovaerts, F. de Vylder and J. Haezendonck, Insurance Premiums. Amsterdam: North-Holland, 1984

[12] G. K. Janssens and K. M. Ramaekers, "On the use of bounds for the stoploss premium for an inventory decision problem", Journal of Interdisciplinary Mathematics, vol. 11, no. 1, pp. 115-126, 2008 https://doi.org/10.1080/09720502.2008.10700546

Gerrit K. Janssens received degrees of $M . S c$. in Business Engineering from the University of Antwerp (RUCA), Belgium, M. Sc. in Computer Science from the University of Ghent (RUG), Belgium, and Dr. sc. ing. in Computer Science in 1989 from the Free University of Brussels (VUB), Belgium. After some years of work at General Motors Continental, Antwerp, he joined the University of Antwerp.

He was a Professor of Operations Management and Logistics at Hasselt University (UHasselt) within the Faculty of Business Economics from 2000 to 2016. He is now Emeritus Professor within the Research Group Logistics. He was the President of the Belgian Operations Research Society (ORBEL) in the period of 2006-2007. During the past twenty-nine years, he has been severa times visiting universities in South-East Asia and Africa. His main research interests include the development and application of operations research models in production and distribution logistics.

Address: Faculty of Business Economics, Research group Logistics Agoralaan 1, B-3590 Diepenbeek, Belgium.

E-mail: gerrit.janssens@uhasselt.be
Lotte Verdonck received the degree of M.Sc. in Business Engineering in 2011 from Hasselt University. From 2011 to 2012, she worked as a Research Assistant on a project sponsored by Hasselt University. From 2012 to 2017, she was a scholar for the FWO (Flemish Fund for Scientific Research). In May 2017 she obtained a PhD degree in Business Economics from the Hasselt University. From then on she has been employed on a post-doctoral position at Hasselt University focusing on horizontal logistics collaboration.

Address: Faculty of Business Economics, Research group Logistics Agoralaan 1, B-3590 Diepenbeek, Belgium.

E-mail: lotte.verdonck@uhasselt.be

Katrien Ramaekers has been Assistant Professor in Operations Management and Logistics at the Faculty of Business Economics (BEW) of Hasselt University since October 2014. She obtained her PhD in Applied Economics in 2007 at Hasselt University.

After obtaining her $\mathrm{PhD}$, she first worked a few years at the Transportation Research Institute of Hasselt University where she took part in several applied research projects of the institute. From 2009 to 2014, she was a Postdoctoral Assistant at the Faculty of Business Economics of Hasselt University. Her research interest goes to the application of operations research techniques in the field of operations management and logistics, with a strong focus on simulation (optimization). In her $\mathrm{PhD}$, she developed a simulation optimization framework for inventory management decision support based on incomplete information. Address: Faculty of Business Economics, Research group Logistics Agoralaan 1, B-3590 Diepenbeek, Belgium.

E-mail: katrien.ramaekers@uhasselt.be 